\title{
Different Response Kinetics to Temperature and Water Vapor of Acrylamide Polymers Obtained by Initiated Chemical Vapor Deposition
}

\author{
Paul Salzmann, Alberto Perrotta, ${ }^{(0)}$ and Anna Maria Coclite*(i) \\ Institute of Solid State Physics, Graz University of Technology, Petersgasse 16, 8010 Graz, Austria
}

Supporting Information

ABSTRACT: Thermoresponsive polymers undergo a reversible phase transition at their lower critical solution temperature (LCST) from a hydrated hydrophilic state at temperatures below the LCST to a collapsed hydrophobic state at higher temperatures. This results in a strong response to temperature when in aqueous environment. This study shows that hydrogel thin films synthesized by initiated chemical vapor deposition show fast and strong response to temperature also in water vapor environment. Thin films of cross-linked poly(N-isopropylacrylamide), $\mathrm{p}(\mathrm{NIPAAm})$, were found to

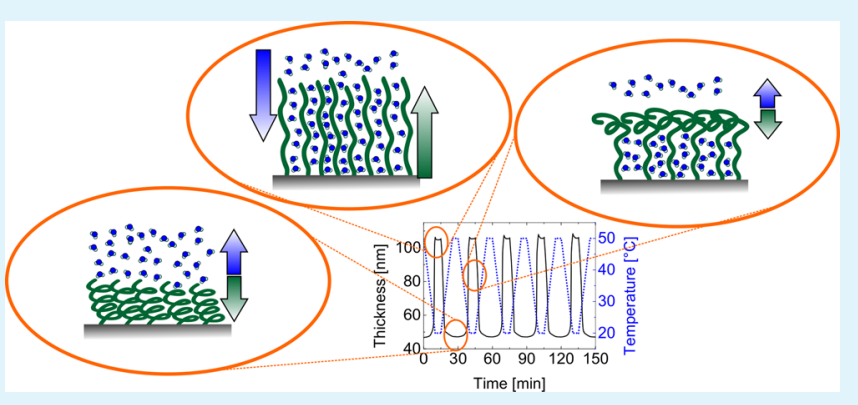
have a sharp change in thickness by $200 \%$ in water vapor at temperatures above and below the LCST. Additionally, the stimuli-responsive poly $(N, N$-diethylacrylamide $)$ was investigated and compared to results found for $\mathrm{p}$ (NIPAAm). Analysis of the swelling kinetics performed with in situ spectroscopic ellipsometry with variable stage temperature shows differences for swelling and deswelling processes, and a hysteresis in the thickness profile was found as a function of temperature and of temperature change rate.

KEYWORDS: NIPAAm, thermoresponsive polymers, iCVD, ellipsometry, swelling kinetics, thin films

\section{INTRODUCTION}

Smart stimuli-responsive artificial hydrogels are water insoluble polymer networks, which respond to stimuli in their environment, for example, temperature, $\mathrm{pH}$, and light. These hydrogels have found applications in a variety of technological fields, such as sensors ${ }^{1}$ and actuators, ${ }^{2}$ controlled drug delivery, ${ }^{3}$ or as potential materials for artificial muscle tissue. ${ }^{4}$ The main requirement for this class of materials is to achieve a large response amplitude and fast response to a small stimulus.

Poly $(N$-isopropylacrylamide $)(\mathrm{p}(\mathrm{NIPAAm}))$ is one of the most prominent representatives of thermoresponsive polymers because its temperature sensitivity is close to body temperature, therefore suitable for biomedical applications such as selective drug release and smart cell culture surfaces. ${ }^{5}$ It undergoes a large reversible volume change ${ }^{6}$ when the so-called lower critical solution temperature (LCST) is reached. ${ }^{7}$ For bulk pure $\mathrm{p}$ (NIPAAm), the LCST is reported at $32{ }^{\circ} \mathrm{C}$. ${ }^{8}$ Below this temperature, the polymer shows hydrophilicity and swells upon water exposure. When the polymer is instead heated at temperatures above the LCST, a phase transition to a hydrophobic state takes place, causing the collapse of the polymer backbone because of the formation of chain-to-chain interactions, eventually leading to expulsion of water and shrinking. ${ }^{9}$

When implemented in sensors and actuators, the swelling behavior of the stimuli-responsive films is a crucial parameter. The dynamics and kinetics of the swelling and deswelling processes determine the response time to an external stimulus, directly influencing the sensing properties. The study of the swelling mechanisms becomes therefore paramount when the engineering of sensing devices and new combinations of polymers is investigated. In the present study, the swelling of two different cross-linked thermoresponsive polymer thin films, namely $\mathrm{p}$ (NIPAAm) and polydiethylacrylamide $\mathrm{p}(\mathrm{DEEAm})$, was investigated, and insights into the kinetics of the thermoresponsive behavior were given to shed light on the applicability of such systems in sensing devices. Examples of NIPAAm-based devices are present in the literature. Ramakrishnan et al. built a humidity sensor using NIPAAm nanogels as the sensing material ${ }^{1}$ in surface acoustic wave devices, and they showed promising results in sensing different humidity levels. However, no information is given about response time and kinetics. To achieve sensors with desired time resolution and to tailor the materials for specific applications, a deeper understanding of swelling and deswelling mechanism is necessary.

The swelling kinetic processes of $p$ (NIPAAm) when in contact with liquid water are well-documented in the literature and have been investigated in previous studies in diverse systems and combinations, for example, spherical gels or thin

Received: December 12, 2017

Accepted: January 26, 2018

Published: January 27, 2018 
films. ${ }^{10-13}$ All of the studies reported on complex and nonhomogeneous mechanisms of swelling and deswelling, dependent on the polymer chemical composition, as well as the swelling conditions. Core-shell p(NIPAAm) nanoparticles were investigated by Lyon. ${ }^{12}$ The nanoparticles were made of both poly(NIPAAm-co-acrylic acid) core and $\mathrm{p}$ (NIPAAm) shell and vice versa. The study showed $\mathrm{pH}$ - and temperaturedependent multistep swelling. Park and Hoffman ${ }^{14}$ reported that the water content of the hydrogel above the LCST was dependent upon the previous state of the gel, the heating rate, and the film thickness, pointing out a complex kinetic process to account for when applying these materials for sensing applications. For deswelling kinetic processes, caffeine-releasing experiments indicated a nonhomogeneous water release, leading to the formation of water pockets within the hydrogel matrix, which delayed the deswelling. These water pockets support the concept of the formation of a dense region at the vapor/film interface during the collapse of $\mathrm{p}$ (NIPAAm), leading to a retarded water release.

Thermodynamic investigations of NIPAAm hydrogels showed that the deswelling process upon heating occurs in two stages. In the first stage, the phase transition begins with the loss of interactions between polymer hydrophobic segments. ${ }^{15}$ In the following stage, the intramolecular hydrogen bonds break and the polymer network converts to a globule form, leading to a dramatic change in volume.

Contrary to the aforementioned studies, which focused on materials in liquid water, in many applications, the NIPAAmbased polymers are not in direct contact with bulk water, and for this reason, studying the swelling behavior in water vapor is crucial to investigate the material properties in its application environment. In the present investigation, the swelling behavior of the two different thermoresponsive polymer thin films, synthesized by initiated chemical vapor deposition (iCVD), was investigated by spectroscopic ellipsometry in liquid water and upon water vapor exposure at different temperatures, having in mind an application of such hydrogels as sensors or actuators in contact with water vapor. In the literature, few examples are reported where the thermoresponsive systems were studied in water vapor. Secrist and Nolte investigated the swelling of a polyelectrolyte multilayer (PEM) of poly(allylamine hydrochloride) and poly(acrylic acid) in humidity using in situ reflectivity. ${ }^{16}$ Their kinetic swelling measurements show that the structural response to humidity can occur in two distinct regimes, similar to what was found in liquid water for NIPAAmderived films: a first initial response on the order of seconds, followed by a longer time scale structural relaxation on the order of hours to days. Because of this, a significant swelling/ deswelling hysteresis was found because the PEM seems to retain some "memory" of its humidity exposure regarding the internal structure.

Synthesis of smart responsive polymer thin films via iCVD has been previously achieved, ${ }^{17-21}$ and it was demonstrated that the technique allows the total retention of the monomer functional groups, essential for engineering smart materials with specific stimuli responses. ${ }^{17}$ Furthermore, low process temperatures can be adopted, allowing the treatment of thermalsensitive substrates. The free radicals initiating the polymerization are obtained by the thermal decomposition of a radical initiator. $^{18}$ Functional monomer units and the sacrificial initiator molecules are introduced into the reactor chamber where heated filament wires $\left(<300{ }^{\circ} \mathrm{C}\right)^{22}$ break the initiator molecules and form radicals, whereas the monomer structure is retained. When the monomer units and the initiator radicals both adsorb at the cooled substrate surface, polymerization is initiated. $^{23}$

We aimed at clearly showing the dependence between the swelling and deswelling processes on the heating/cooling rate. In particular, we chose to use fast temperature change rates (from 6 to $3{ }^{\circ} \mathrm{C} / \mathrm{min}$ ) at which the hydrogel was not completely thermalized, and therefore in a nonequilibrium state, which we retain closer to real-world test conditions.

\section{EXPERIMENTAL SECTION}

Thin Film Synthesis. Copolymer thin films of NIPAAm $(\geq 99 \%$, Sigma-Aldrich, Germany) and N,N-diethylacrylamide (DEAAm, 99\%, Sigma-Aldrich, Germany) cross-linked with di(ethylene glycol) divinyl ether (DEGDVE, 99\%, Sigma-Aldrich, Germany) were synthesized using a custom-built iCVD reactor. As radical initiator, tert-butyl peroxide (TBPO, 98\%, Sigma-Aldrich, Germany) was used in the process. NIPAAm, DEAAm, and DEGDVE were kept at 85,80 , and 70 ${ }^{\circ} \mathrm{C}$, respectively, and flown in the reactor through heated lines (100 ${ }^{\circ} \mathrm{C}$ ) to avoid condensation. The initiator TBPO was kept at room temperature and fed in the reactor through a separate line. To control the chemical composition of the thin films, the flow rates of the chemicals were controlled by needle valves. The flow rates of the monomers varied in the range of $0.5-0.2$ and $2.9-0.5 \mathrm{sccm}$ for NIPAAm and DEGDVE, respectively, when depositing NIPAAmbased polymers; for DEAAm-based polymers, the flow rates varied in the range of $0.6-0.3$ and $0.9-0.5 \mathrm{sccm}$ for the DEAAm and DEGDVE, respectively. The initiator flow rate varied in the range of 1.1-0.4 $\mathrm{sccm}$. The working pressure in the reactor chamber was $250 \mathrm{mT}$ Torr for all depositions. The substrate temperature was set to $35 \pm 2{ }^{\circ} \mathrm{C}$. As soon as the working pressure was reached, the filament wires were heated to $220{ }^{\circ} \mathrm{C}$ to initiate thermal decomposition of the TBPO molecules, starting the deposition process. All films were deposited on crystalline $(\langle 100\rangle)$ silicon wafers (SIEGERT WAFER GmbH) with native silicon oxide layers. Laser interferometry $(\mathrm{He}-\mathrm{Ne}$ Laser with $\lambda$ $=633 \mathrm{~nm}$, Thorlabs) was used for in situ growth monitoring. All of the studied films have a thickness in the range of $50-200 \mathrm{~nm}$.

Characterization. For chemical analysis of the obtained thin films, Fourier-transform infrared (FTIR) spectroscopy (Bruker IFS 66v/S) was performed. For each spectrum, 1000 scans were recorded in the transmission mode with a resolution of $4 \mathrm{~cm}^{-1}$ between 400 and 4000 $\mathrm{cm}^{-1}$. All spectra were baseline-corrected and normalized to the film thickness. The characteristic absorption of the $\mathrm{C}=\mathrm{O}$ stretch vibrational mode for amides $\left(1640-1690 \mathrm{~cm}^{-1}\right.$ ) was used to qualitatively estimate the DEGDVE fraction in the copolymer. ${ }^{24}$ Film thickness measurements and swelling experiments in liquid water and humid air environment were carried out with (in situ) spectroscopic ellipsometry (J. A. Woollam, M-2000). Spectra with wavelengths between 370 and $1000 \mathrm{~nm}$ were recorded. Accurate thickness determination was performed in a three-angle measurement $\left(65^{\circ}, 70^{\circ}\right.$, and $\left.75^{\circ}\right)$. For in situ swelling experiments in liquid water, a setup with a liquid cell ( $5 \mathrm{~mL}$ in volume, J. A. Woollam) was used to measure the films in deionized water $\left(T=25{ }^{\circ} \mathrm{C}\right)$. In this setup, the incident angle was fixed to $75^{\circ}$. For in situ measurements in humid air, a temperature stage (THMS600, Linkam) capped with a sealing chamber was used, where the temperature can be precisely controlled. Elevated relative humidity $(>85 \%)$ was obtained with a homemade pumping system, allowing for a constant flow of humid air in the chamber.

The data measured in dry conditions were modeled with a threelayer approach comprised of $\mathrm{Si}$ substrate, native silicon oxide layer $(\sim 1.7 \mathrm{~nm})$, and a Cauchy layer to model the transparent polymer layer, defined as

$$
n(\lambda)=A+\frac{B}{\lambda}+\frac{C}{\lambda^{2}}
$$

where $n$ is the refractive index of the investigated film; $A, B$, and $C$ are constants; and $\lambda$ is the light wavelength. 
a)

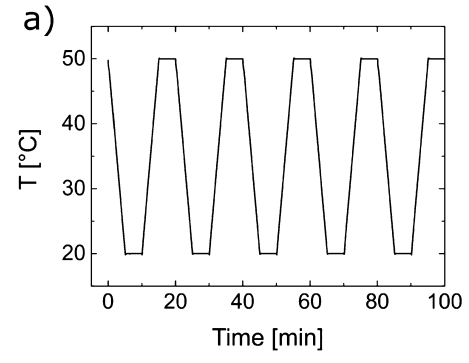

b)

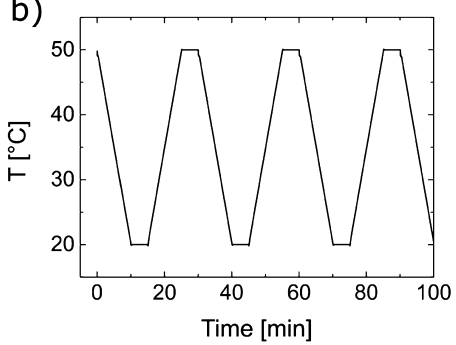

c)

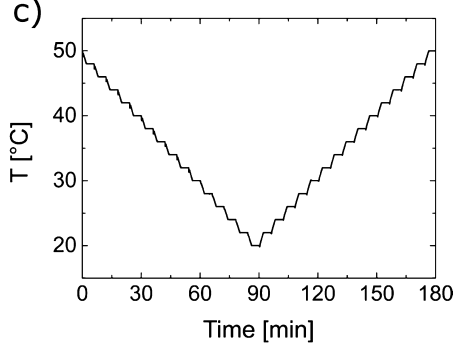

Figure 1. Different temperature ramps. (a) $\mathrm{T}$ profile 1: The temperature was ramped from 50 to $20{ }^{\circ} \mathrm{C}$ within 5 min and held constant for 5 min, before ramping back from 20 to $50^{\circ} \mathrm{C}$. (b) $T$ profile 2: The temperature was ramped within $10 \mathrm{~min}$ and hold constant for 5 min between ramps. (c) $T$ profile 3: In this case, the temperature was ramped from $50{ }^{\circ} \mathrm{C}$ by $2{ }^{\circ} \mathrm{C}$ within 2 min and hold constant between these $2{ }^{\circ} \mathrm{C}$ steps for 2 more minutes until the temperature reached $20^{\circ} \mathrm{C}$. When $T$ arrived $20^{\circ} \mathrm{C}$, the system was heated in the same pattern up to $50{ }^{\circ} \mathrm{C}$.
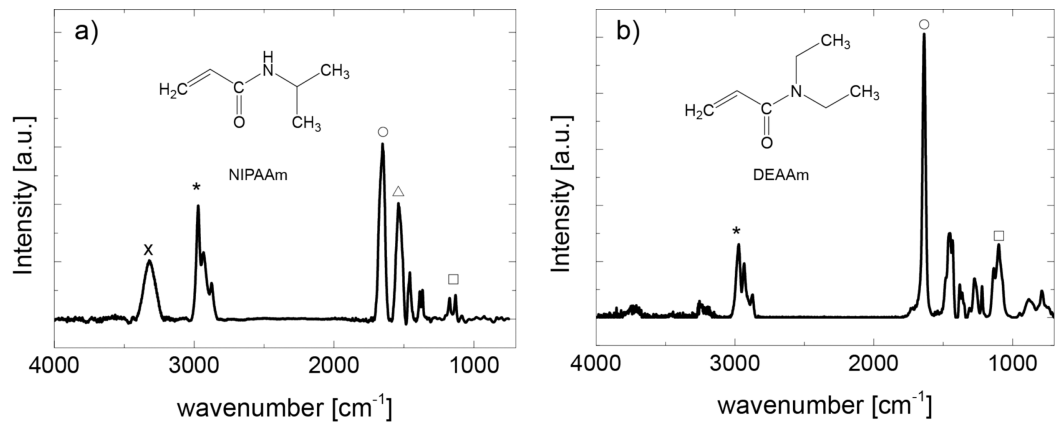

Figure 2. (a) FTIR spectrum of a p(NIPAAm-co-DEGDVE) sample $(50 \mathrm{~nm})$ (b) Spectrum of a p(DEAAm-co-DEGDVE) sample (50 nm). The adsorptions between 3000 and $2820 \mathrm{~cm}^{-1}$ can be attributed to the $\mathrm{C}-\mathrm{H}_{x}(x=2,3)$ stretching bands $(*)$, the peak at $1645 \mathrm{~cm}{ }^{-1}$ to the $\mathrm{C}=\mathrm{O}$ stretching in the amide groups $(\mathrm{O})$. The characteristic bands of the secondary amide groups are visible for the $\mathrm{N}-\mathrm{H}$ stretching at $3500-3000 \mathrm{~cm}^{-1}$ $(\times), \mathrm{N}-\mathrm{H}$ bending at $1500 \mathrm{~cm}^{-1}(\Delta)$, and $\mathrm{C}-\mathrm{N}$ stretching at $1150 \mathrm{~cm}^{-1}(\square)$.

The layers were put in contact with the liquid water, and the increase of thickness upon water exposure was investigated. In the presence of water (liquid and vapor), an effective medium approximation layer was adopted, treating the polymer film as a mixture of two constituent materials with defined optical constants (water and polymer layer modeled by a Cauchy layer with optical constants obtained in dry-state measurements). The swelling ratio, $\Delta t$, is defined as

$$
\Delta t=\frac{t_{\mathrm{wet}}-t_{\mathrm{dry}}}{t_{\mathrm{dry}}}
$$

where $t_{\text {wet }}$ is the thickness of the swollen hydrogel in the presence of water and $t_{\mathrm{dry}}$ is the initial thickness of the hydrogel in atmosphere.

For the swelling experiments of $\mathrm{p}$ (NIPAAm-co-DEGDVE) and $\mathrm{p}$ (DEAAm-co-DEGDVE) films in humid air, the following procedure was followed. At first, the sample stage was heated to $50{ }^{\circ} \mathrm{C}$ in air, and the thickness was measured in situ until it reached the equilibrium steady-state value $(5 \mathrm{~min})$. In the next step, the humidity pump was turned on whereas the stage temperature was kept constant at $50{ }^{\circ} \mathrm{C}$. The relative humidity was monitored via a sensor in the measurement chamber (Sparkfun SHT-15). When the relative humidity and the thickness reached equilibrium, the actual in situ measurements in humid air with cycling stage temperature between 50 and $20{ }^{\circ} \mathrm{C}$ were performed. Three different temperature ramps were used (Figure 1). When profile 1 was applied (Figure 1a), the temperature was ramped from 50 to $20^{\circ} \mathrm{C}$ and vice versa within $5 \mathrm{~min}\left(6^{\circ} \mathrm{C} / \mathrm{min}\right)$, holding the temperature constant for $5 \mathrm{~min}$ between the ramps. Using profile 2 (Figure $1 \mathrm{~b})$, the ramping time was $10 \mathrm{~min}\left(3^{\circ} \mathrm{C} / \mathrm{min}\right)$, with again 5 min of holding the temperature constant in between. The temperature was cycled five times in all experiments. The temperature profiles were chosen to investigate the system in conditions mimicking a real sensor/actuator application, that is, nonequilibrium conditions. To get deeper insights into the swelling kinetics, a third type of temperature ramp experiment was performed (Figure 1c). The temperature was ramped stepwise from 50 to $20^{\circ} \mathrm{C}$ and vice versa by $2{ }^{\circ} \mathrm{C}$, holding the temperature constant for 2 minutes between the steps $\left(0.33^{\circ} \mathrm{C} / \mathrm{min}\right)$. The time resolution in all in situ experiments was $2 \mathrm{~s}$.

\section{RESULTS AND DISCUSSION}

Chemical Composition. The stimuli-responsive p(NIPAAm-co-DEGDVE) and p(DEAAm-co-DEGDVE) polymer thin films with different monomer ratios were synthetized by iCVD. FTIR spectroscopy was used for the chemical characterization of the polymers. The areas of the $\mathrm{C}=\mathrm{O}$ stretching bands $\left(\sim 1650 \mathrm{~cm}^{-1}\right)$ were qualitatively compared to the adsorbed monomer fractions $f$ calculated as

$$
f_{\mathrm{A}}=\frac{\left(\frac{P_{\mathrm{m}}}{P_{\text {sat }}}\right)_{\mathrm{A}}}{\left(\frac{P_{\mathrm{m}}}{P_{\text {sat }}}\right)_{\mathrm{A}}+\left(\frac{P_{\mathrm{m}}}{P_{\text {sat }}}\right)_{\mathrm{B}}} \%
$$

where $\mathrm{A}$ and $\mathrm{B}$ indicate two generic monomers and $P_{\mathrm{m}} / P_{\text {sat }}$ is the ratio of the partial pressure of the monomer $P_{\mathrm{m}}$, and its saturation pressure $P_{\text {sat }}$ of the monomer at the substrate temperature. ${ }^{a} P_{\mathrm{m}} / P_{\text {sat }}$ values were set prior to the deposition of the films and determine the concentration of monomer adsorbed on the surface, the growth rate, and the degree of conformality. $^{23}$ The spectra shown in Figure 2 point out a successful polymerization with full retention of the monomer functional groups. The strong adsorptions between 3000 and $2820 \mathrm{~cm}^{-1}$ can be attributed to the $\mathrm{C}-\mathrm{H}_{x}(x=2,3)$ stretching bands, whereas the peak at $1645 \mathrm{~cm}^{-1}$ to the $\mathrm{C}=\mathrm{O}$ stretching in the amide groups. The lack of characteristic bands corresponding to the vinyl groups typically at 1620, 1400, and $3150 \mathrm{~cm}^{-1}$ points out that the polymerizations were successful. The presence of cross-linker units could not be 

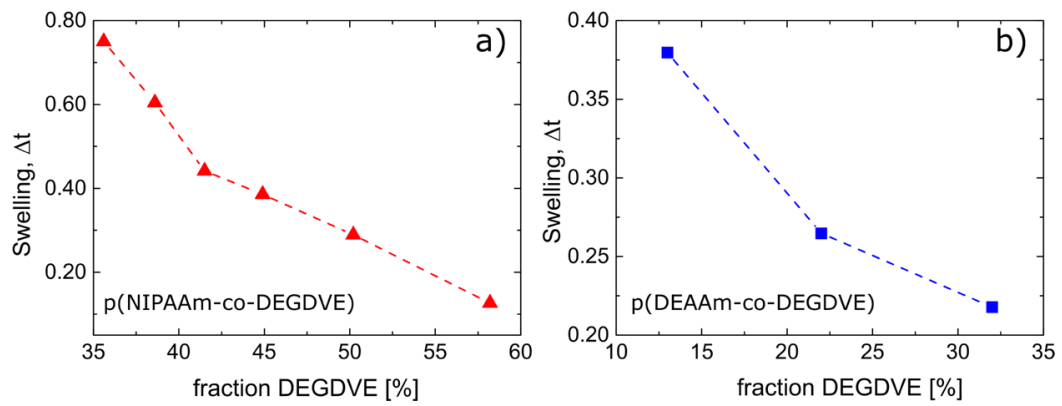

Figure 3. Swelling ratio $(\Delta t)$ for the copolymers (a) $\mathrm{p}$ (NIPAAm-co-DEGDVE) and (b) $\mathrm{p}$ (DEAAm-co-DEGDVE) prepared with different compositions, calculated via the $P_{\mathrm{m}} / P_{\text {sat }}$ of the DEGDVE. At elevated DEGDVE ratios, the swelling ratio decreases. The error on the $\Delta t$ is \pm 0.05 . The error on the DEGDVE content is $5 \%$. These errors are calculated from the variations of thickness and composition measured in every single batch of samples. The DEGDVE fractions are intended as the available cross-linker molecules on the surface during the deposition and are related to, but do not correspond to, the amount of cross-linker included in the polymer.
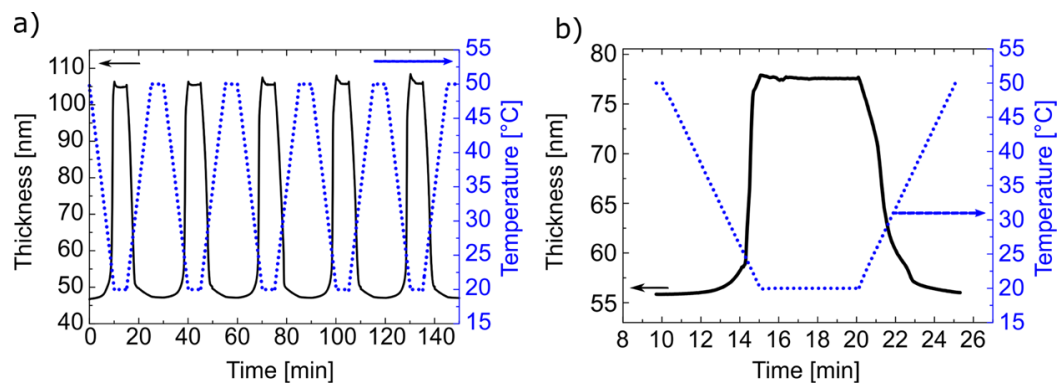

Figure 4. (a) In situ swelling experiment on p(NIPAAm-co-DEGDVE) in humid air environment with cycling stage temperature (profile 1). The fitted film thickness increases as the temperature decreases and vice versa. (b) Detail of a temperature profile 1 in situ ellipsometry measurement performed on p(DEAAm-co-DEGDVE).

confirmed by FTIR because of the weak FTIR absorption bands attributed to DEGDVE. The characteristic bands of the secondary amide groups were also present: $\mathrm{N}-\mathrm{H}$ stretching at $3500-3000 \mathrm{~cm}^{-1}, \mathrm{~N}-\mathrm{H}$ bending at $1500 \mathrm{~cm}^{-1}$, and $\mathrm{C}-\mathrm{N}$ stretching at $1150 \mathrm{~cm}^{-1}$.

The presence of cross-linker in the polymer was verified by swelling experiments in liquid water measured by spectroscopic ellipsometry. The ratio of the functional monomer, NIPAAm or DEAAm, and cross-linker DEGDVE in the film was found to strongly affect the swelling in water at room temperature. Increasing the amount of cross-linking units leads to a decrease in the swelling ratio because of the fewer hydrophilic functional groups and the higher density and rigidity introduced by the cross-linker, confirming the successful copolymerization. ${ }^{23}$ Figure 3 shows the swelling ratios for different amount of DEGDVE in the $\mathrm{p}$ (NIPAAm-co-DEGDVE) and $\mathrm{p}$ (DEAAm-coDEGDVE) thin films, respectively, as a function of the $P_{\mathrm{m}} / P_{\text {sat }}$ ratios. $\mathrm{p}$ (NIPAAm-co-DEGDVE) films with less than $35 \%$ DEGDVE were not stable in liquid water. Samples prepared with $\mathrm{p}$ (DEAAm-co-DEGDVE) were stable with DEGDVE ratios less than $30 \%$ because $\mathrm{p}(\mathrm{DEAAm})$ can take up less water compared to $\mathrm{p}$ (NIPAAm). Schmaljohann et al. suggest that $\mathrm{p}$ (DEAAm) can only act as the hydrogen bond acceptor because of the lack of NH moieties, whereas $p$ (NIPAAm) can also act as hydrogen bond donor. ${ }^{25}$ Therefore, $p$ (DEAAm) is stable in water also with less cross-linking.

Swelling Behavior in Water Vapor. For the experiments in water vapor, the samples that showed stability in water with the minimum amount of cross-linker were chosen (i.e., $\mathrm{p}$ (NIPAAm-co-DEGDVE) sample with 35\% DEGDVE and $\mathrm{p}$ (DEAAm-co-DEGDVE) with 15\% DEGDVE). Even though the samples that dissolve in water would be stable in water vapor and allow for a larger swelling response, by choosing a lower cross-linker ratio, we could not have compared the response in water and water vapor. Figure $4 a$ shows the data collected in an in situ ellipsometry measurement in water vapor with cycling stage temperature on the p(NIPAAm-coDEGDVE) sample with 35\% DEGDVE.

At the beginning, when the stage temperature is at $50{ }^{\circ} \mathrm{C}$, the thickness of the thermoresponsive polymer is close to the dry state, with some residual water in the system $(10 \%$ increase compared to dry films). At $50{ }^{\circ} \mathrm{C}$, the polymer is in a hydrophobic state in which the polymer network forms interchain hydrogen bonds and allows limited water molecules to be absorbed. When the temperature decreases below the LCST, the material undergoes a reversible phase transition to a hydrophilic state, taking up water from the humid environment, which leads to an increase of $120 \%$ in film thickness. By increasing the temperature to $50{ }^{\circ} \mathrm{C}$, the polymer expels the uptaken water, shrinking to its original thickness. This behavior can be observed in all five repeating cycles of the experiment. The reversible transition between swollen and dehydrated state occurs within a few seconds, pointing out a very fast response to temperature variations. In the literature, different studies found that the deswelling process for $\mathrm{p}$ (NIPAAm) hydrogels is slower, with recovering times on the order of several hours up to a month to reach the equilibrium deswelling state. ${ }^{13}$ On the other hand, Okano et al. ${ }^{13}$ studied comb-type crafted hydrogels of NIPAAm. The study shows that the swelling and deswelling of comb-grafted hydrogels shows a fast response, similar to the one also observed for the iCVD polymers, and dependent on the graft chain lengths.

In a similar fashion, $p$ (DEAAm-co-DEGDVE) films were analyzed (Figure 4b). The thickness increase was 100\% 

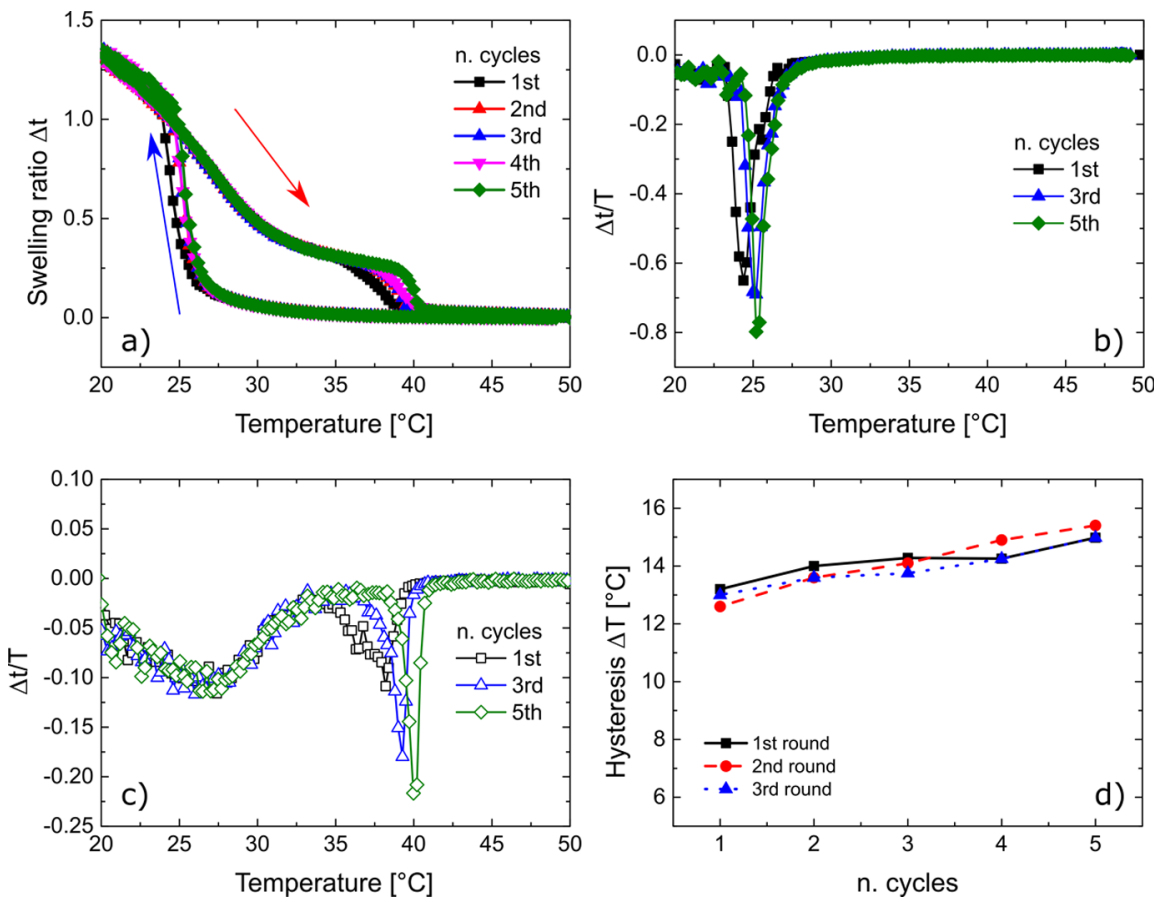

Figure 5. (a) Swelling ratio at different temperatures obtained from in situ ellipsometry measurements on p(NIPAAm-co-DEGDVE) films (50 nm). Temperature profile 1 was ramped in this measurement. (b) First derivative of swelling data with respect to temperature plotted against temperature for the cooling ramp. The plot in (c) shows the first derivative of the swelling ratio with respect to the temperature for the heating ramp. The temperatures, at which the change in thickness is the highest (i.e., the LCST) differ for cooling and heating. (d) Hysteresis $\Delta T$ as a function of the number of cycles for $\mathrm{p}$ (NIPAAm-co-DEGDVE). The hysteresis is calculated between the temperatures of the fastest temperature change rate in heating and cooling, respectively. The sample was dried, and the experiment was repeated other two times (round 2 and round 3 ).

compared to the thickness at higher temperature (dehydrated state). The lower swelling degree of $\mathrm{p}$ (DEAAm-co-DEGDVE) compared to $\mathrm{p}$ (NIPAAm-co-DEGDVE) is probably because of the lack of $\mathrm{NH}$ moieties, ${ }^{21}$ which limits the formation of intermolecular hydrogen bonds with water. As for the NIPAAm-based polymer, the p(DEAAm-co-DEGDVE) shows fast response and reproducibility of the swelling/deswelling cycles (data not shown).

It is worth noticing that the spike in measured thickness at the beginning of every temperature cycle when the system arrives to the hydrophilic swollen state, as shown in Figure 4a, may be caused by an overshoot in temperature, for example, the temperature reaches values slightly below $20{ }^{\circ} \mathrm{C}$ for a short period of time. Another possible explanation is an elastic effect, that is, the film thickness reaches, out of equilibrium, higher values when water is absorbed and relaxes back to an equilibrium value. For samples prepared with DEAAm, this effect is smaller, probably because of the lower intrinsic swelling, and, in turn, lower water uptake.

Swelling Kinetics. To interpret the swelling behaviors and determine the transition temperatures (LCSTs), the data of Figure 4 were replotted as swelling ratio as a function of temperature (Figure 5a) for the five cycles performed on the $\mathrm{p}$ (NIPAAm-co-DEGDVE) layers.

The swelling ratio varied from 1.3 to 0.1 when the temperature was cycled between 20 and $50{ }^{\circ} \mathrm{C}$, respectively. A hysteresis was found between the heating and cooling cycles. The temperature at which the transition between a hydrophobic and a hydrophilic state takes place strongly differs for heating and cooling. During heating, the swelling ratio was found to decrease at a slower rate compared to the same transition occurring during cooling. Furthermore, a step-like mechanism was found. The shrinking reaches a plateau at 33 ${ }^{\circ} \mathrm{C}$, with the thickness variation on the order of less than $10 \%$. For the first cycle, a second sharper variation in thickness was found to occur at $36{ }^{\circ} \mathrm{C}$. Such a clear step-like shrinking was never reported before in response to humidity, whereas it was observed in water. ${ }^{10}$ The step-like shrinking hints at the presence of different deswelling mechanisms as the temperature increases. In the literature, the formation of a denser collapsed skin-like top layer at the film surface is hypothesized for NIPAAm-containing polymers for swelling/deswelling in liquid water. $^{10-14,26,27}$ The step-like mechanism can be then attributed to the formation of a denser top layer. Around 36 ${ }^{\circ} \mathrm{C}$, the residual water molecules trapped in the film have enough energy to diffuse through the denser skin-like layer and leave the polymer network, resulting in the second sharp transition. Such step-like process of deswelling was observed also by Matsuo and Tanaka on spherical gel bodies of $\mathrm{p}$ (NIPAAm). ${ }^{10}$ The authors stated that deswelling is more complex than the swelling process. Three different time domains were identified during heating with two deswelling periods alternated with a plateau, where no or very little variation in thickness is measured. ${ }^{10}$ The plateau was ascribed to the formation of a thick layer of dense, collapsed polymer network on the surface of the polymer, considered impermeable to the inner remaining water and preventing a further shrinking. At the end of the plateau region, bubbles appear at the surface, which then disappear when the gel bodies shrink further. They suggest that these patterns result from mechanical and thermodynamic instabilities. These three time domains are similar to what is reported in Figure 5a. The three time domains can be qualitatively described as follows. In the first period, the swollen hydrogel starts to shrink locally in a thin 

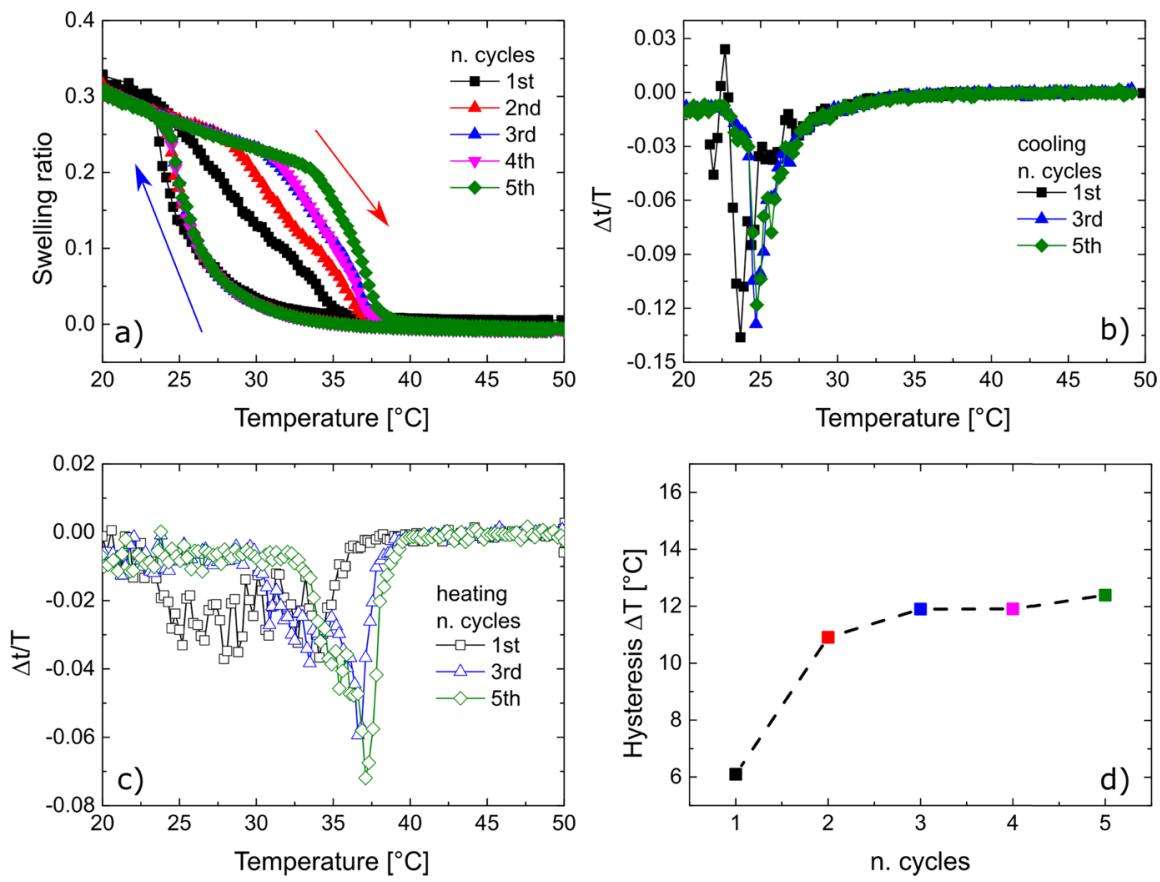

Figure 6. (a) p(DEAAm-co-DEGDVE) swelling kinetics. Temperature profile 1 was ramped in this measurement. Two slopes are visible, indicating a two-step deswelling process, as found for $\mathrm{p}$ (NIPAAm). (b) First derivative of swelling with respect to temperature for the cooling ramp plotted against temperature. (c) First derivative of the swelling ratio with respect to the temperature for the heating ramp. (d) Hysteresis $\Delta T$ as a function of the number of cycles for $\mathrm{p}$ (DEAAm-co-DEGDVE). The hysteresis is calculated between the temperatures of the fastest temperature change rate in heating and cooling, respectively.

surface region only. This collapsed dense polymer network layer works as a barrier for the water still present in the polymer and prevents further shrinking, resulting in a plateau. When the osmotic pressure in the inner sphere gets sufficiently high, the surface layer is then permeable again and the film continues to shrink. In the Matsuo and Tanaka paper, the first plateau was observed at lower temperatures than in our system. We believe this is a consequence of the DEGDVE additional cross-linking, which creates-in our case-a denser skin layer compared to what happens to the linear polymer chains. In a previous study, we reported some lines of evidence for the formation of a glassy skin at a thin polymer film-air interface at low humidity $(<75 \%)$ when studying hydrogels because of the high Flory interaction parameter at these conditions. ${ }^{27}$ The formation of a dense skin with underlying water pockets for the deswelling process and a dependency of water uptake on the previous hydration state was also found by Park and Hoffmann in aqueous solution. ${ }^{14}$ Fast dynamic and slow equilibrium swelling was also reported in another study. ${ }^{25}$

For the cooling process, instead, the swelling was found to occur in a very small temperature range between $\sim 27$ and $\sim 24$ ${ }^{\circ} \mathrm{C}$. A sharp increase in polymer thickness clearly indicates a single-dominant swelling mechanism. It is worth noticing that the thickness was found to vary also between 20 and $25{ }^{\circ} \mathrm{C}$, before the fast swelling process. This variation can be attributed to changes in the adsorption of water at the polymer surface when the temperature varies. Decreasing the temperature from 25 to $20{ }^{\circ} \mathrm{C}$ induces an initial adsorption of water within the polymer matrix, and, in turn, a change in the swelling ratio of 0.2 in the case of the NIPAAm-based polymers investigated in this study. However, this effect has a minor contribution compared to the thermoresponsive rearrangement of the polymer chains, which causes an increase of the swelling ratio of 1 within $2{ }^{\circ} \mathrm{C}$.
To more precisely measure the temperatures at which the thickness variation takes place, the first derivative of the film thickness with respect to the temperature was calculated and plotted as a function of temperature, as reported in Figure 5b,c. From these data, the LCST was defined as the temperature where the highest thickness variation was measured. The data obtained from cooling show one single distinct minimum around $25{ }^{\circ} \mathrm{C}$, irrespective of the number of cycles. For the heating process, instead, two mechanisms can clearly be distinguished: a slow mechanism around $27{ }^{\circ} \mathrm{C}$ and faster mechanism ranging between 37 and $40{ }^{\circ} \mathrm{C}$. The rate of thickness change in the cooling phase is four times higher than in the heating phase, which indicates that the polymer network can rearrange faster when taking up water, compared to shrinking when water leaves the system. The LCST values below $30{ }^{\circ} \mathrm{C}$ are typical for cross-linked NIPAAm. ${ }^{7}$ Additionally, in Figure 5a,c, the deswelling was found to change as a function of the number of cycles. The temperature at which water is released from the polymeric thin film increases with the number of cycles, going from 37 to $40{ }^{\circ} \mathrm{C}$ from the first to the fifth cycle, respectively. The hysteresis $\Delta T$ was calculated as the difference between the temperature of the fastest mechanism during swelling and the one during deswelling and reported in Figure $5 \mathrm{~d}$. As a function of the number of cycles, the hysteresis increases, confirming a different out-diffusion of water for every cycle. The five-cycle swelling/deswelling experiment has been repeated two times after drying the sample, to exclude irreversible changes of the polymeric structure. The hysteresis was reduced when the sample had been dried and was found to increase again in the same fashion as a function of the number of cycles. Other studies on the swelling kinetics of NIPAAm in liquid water described the swelling process of NIPAAm spheres with the ratio of the osmotic bulk modulus of the polymer network to the friction coefficient between the polymer 
network and the solvent and adopting the collective diffusion equation. ${ }^{11}$ They reported a difference in the swelling behavior for the heating and cooling processes, in agreement with our findings. The shrinking process, when the temperature was increased above the LCST, could not be fitted with the diffusion model used. Change in the friction between polymer network and solvent because of the large decrease of hydrogen bonds between water molecules and polymer network could cause these differences in heating and cooling in nonequilibrium conditions.

The swelling kinetics of the $\mathrm{p}$ (DEAAm-co-DEGDVE) thin films were also evaluated. Figure $6 a$ shows the swelling ratio as a function of temperature.

Differences were found in the swelling/deswelling kinetics when compared to the $\mathrm{p}$ (NIPAAm-co-DEGDVE). The response during the cooling process is not as sharp as it was found for $\mathrm{p}$ (NIPAAm-co-DEGDVE). A reason for this slower response may be the lack of $\mathrm{NH}$ moieties and that the hydrogel cannot form intermolecular hydrogen bonds in the dehydrated state. $^{25}$ Therefore, p(DEAAm-co-DEGDVE) polymers cannot transport water in and out of the network as fast as $\mathrm{p}$ (NIPAAmco-DEGDVE) ones. This leads also to the more linear change in thickness for the first shrinking between 20 and $35{ }^{\circ} \mathrm{C}$, compared to the sharper transition seen for $p$ (NIPAAm-coDEGDVE) in Figure 5a. Looking at the data for the first cycle in Figure 6a, the transition was found to happen linearly for almost the whole temperature range. Furthermore, the hysteresis was found to increase from the first to the fifth cycle. Next to the increasing gap between the LCSTs for cooling and heating, the shape of the heating path also changes. While in the first cycle, a slow steady decrease in thickness was found; in the following cycles, a step-like feature appears, reversed with respect to the one observed in the NIPAAmcontaining polymers. A first slow decrease in thickness is followed by an increasingly abrupt drop in the polymer thickness. While for the p(NIPAAm-co-DEGDVE), the step was observed above $35^{\circ} \mathrm{C}$ and for the $\mathrm{p}$ (DEAAm-coDEGDVE), it occurs at lower temperatures. This could be an effect of the lower degree of cross-linking in these systems. The different mechanism of the deswelling process can be better visualized by plotting the rate of thickness change, as shown in Figure $6 \mathrm{~b}, \mathrm{c}$. For the first cycle, no peak in the thickness change rate is visible. With the increasing cycle number, peaks between 35 and $37^{\circ} \mathrm{C}$ become more pronounced, indicating a step-like deswelling mechanism. The differences between $\mathrm{p}$ (DEAAm-coDEGDVE) and $\mathrm{p}$ (NIPAAm-co-DEGDVE) can be attributed to the slower response of DEAAm containing polymers to temperature changes. The formation of the dense collapsed skin layer and rearrangements of chains in the polymer backbone are delayed, as inferred also by the hysteresis increase as a function of the number of cycles (Figure $6 \mathrm{~d}$ ). The DEAAm-containing polymer is found to retain water for a larger temperature range, compared to the NIPAAm-containing polymer, reducing its thickness only of less than $10 \%$ till $35{ }^{\circ} \mathrm{C}$ during the fifth cycle. It is interesting to notice that the hysteresis between swelling and deswelling was associated with the literature to the formation of cross-links in the collapsed state of linear $\mathrm{p}$ (NIPAAm) because of the formation of intramolecular $\mathrm{N}-\mathrm{H}-\mathrm{O}=\mathrm{C} \mathrm{H}$ bonds. ${ }^{28,29}$ This would not justify our observation of a hysteresis also for the DEAAmbased systems that do not have $\mathrm{N}-\mathrm{H}$ groups and therefore the possibility of forming intramolecular $\mathrm{H}$ bonds. Probably, the existing cross-links due to the DEGDVE are the principal factors responsible for hindering the diffusion of water, in this case.

To investigate the nature of the hysteresis, both polymers were studied adopting the temperature profile 2 (Figure 1b), where the temperature is allowed to change slower (ramp time $10 \mathrm{~min}$ ). The hysteresis as a function of the number of cycles is reported in Figure 7.

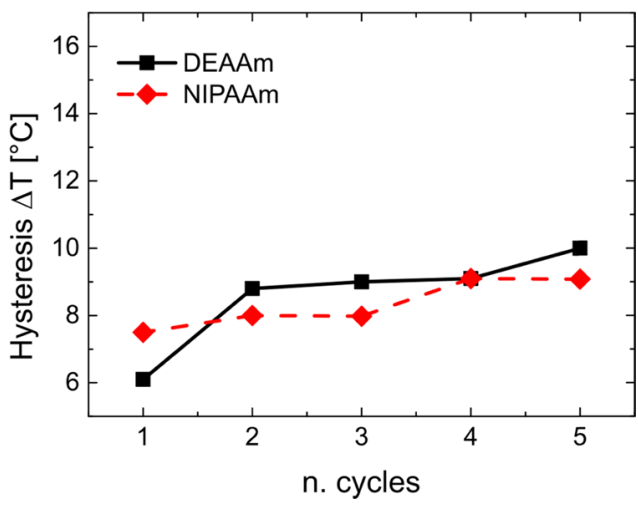

Figure 7. Hysteresis $\Delta T$ as a function of the number of cycles for $\mathrm{p}$ (DEAAm-co-DEGDVE) and $\mathrm{p}$ (NIPAAm-co-DEGDVE). The hysteresis is calculated between the temperatures of the fastest rate of thickness change in heating and cooling, respectively.

Comparing the hysteresis measured adopting profile 1 (Figure 1a), for both polymers, a decrease in the difference in temperature between the cooling and heating branch was found. For $\mathrm{p}$ (NIPAAm-co-DEGDVE), $\Delta T$ was found to decrease by $5-6{ }^{\circ} \mathrm{C}$, whereas for $\mathrm{p}$ (DEAAm-co-DEGDVE) the difference is on the order of $2{ }^{\circ} \mathrm{C}$. The reduction in the hysteresis confirms that this effect stems from the water diffusion mechanism and is related to how the system responds to variations in temperature. When the variation in temperature is slower (profile 2), the deswelling better follows the outdiffusion of water, limiting the formation of a denser outer layer. The larger decrease measured for the NIPAAmcontaining polymers can be attributed again to a faster response in terms of chain rearrangement, as witnessed also in the swelling/deswelling cycles adopting profile 1 .

To verify if the hysteresis is present also in equilibrium conditions, further in situ ellipsometry experiments were performed. In high relative humidity $(>85 \%)$, the temperature was changed from 50 to $20{ }^{\circ} \mathrm{C}$ by $2{ }^{\circ} \mathrm{C}$ steps $2 \mathrm{~min}$ long. The temperature is then kept constant for additional 2 min between every step. When the temperature arrived at $20{ }^{\circ} \mathrm{C}$, the stage was heated up again to $50{ }^{\circ} \mathrm{C}$ in the same fashion. In this way, the system was given enough time to adjust to the change in temperature reaching a constant thickness value for every temperature step. The swelling ratio obtained from this experiment is shown in Figure 8a,b. The temperature profile is plotted in Figure 1c.

Compared to the swelling behavior obtained in the previous experiments, the hysteresis was found to drastically decrease and almost disappear for both polymers. The LCST for swelling and deswelling differs less than $5{ }^{\circ} \mathrm{C}$, and for the deswelling, the response was found sharper. The kinetics for the swelling process during cooling, when the system switches from a hydrophobic to a hydrophilic state, has a similar trend compared to the one measured adopting temperature profile 1 . 

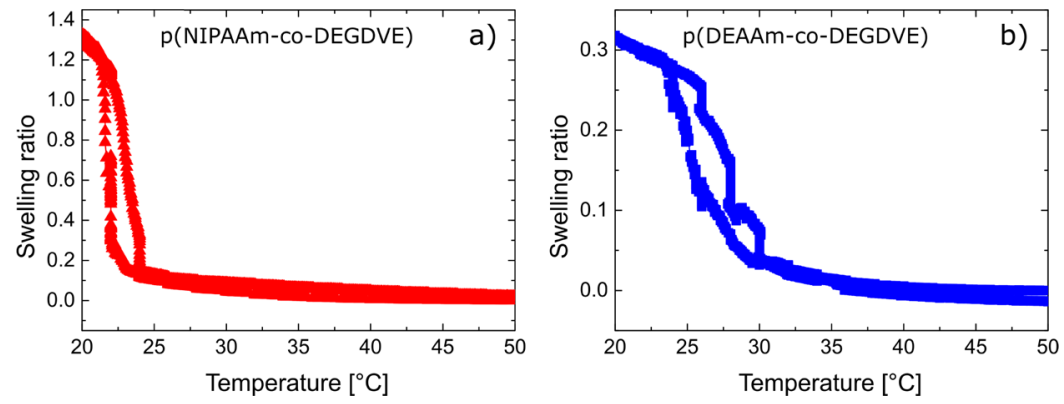

Figure 8. (a) Swelling of a cross-linked $\mathrm{p}$ (NIPAAm-co-DEGDVE) sample at the different temperatures. The hysteresis decreased compared to previous experiments, the LCSTs for swelling and deswelling are within $5{ }^{\circ} \mathrm{C}$. (b) Same experiment was performed with a cross-linked $\mathrm{p}(\mathrm{DEAAm}$-coDEGDVE) thin film.

To summarize, the LCST values observed during the cooling for the $\mathrm{p}$ (NIPAAm) decreases as the temperature rate change decreases, as shown in Table 1.

Table 1. LCST Values for the NIPAAm and DEAAm Containing Polymers Measured with Three Different Temperature Rate Change Profiles

\begin{tabular}{lccc} 
& \multicolumn{3}{c}{ LCST $\left[{ }^{\circ} \mathrm{C}\right]$} \\
\cline { 2 - 4 } monomer & $T$ profile 1 & $T$ profile 2 & $T$ profile 3 \\
NIPAAm & $24.8 \pm 0.5$ & $22.7 \pm 0.5$ & $21.7 \pm 0.5$ \\
DEAAm & $24.7 \pm 0.5$ & $24.7 \pm 0.5$ & $24.8 \pm 0.5$ \\
\hline
\end{tabular}

DEAAm-based polymers did not show any change in the LCST values as a function of the temperature ramp. The differences measured for NIPAAm could be attributed to the faster response of this polymer to temperature changes, affecting its chain rearrangement. This characteristic is crucial when designing sensors based on thin-film NIPAAm-based polymers, as the temperature rate variation should be limited to have a precise response of the thermoresponsive polymer. On the contrary, notwithstanding the slower response to temperature variations and the limited change in volume, DEAAmbased polymers showed a more stable LCST, which makes them more suitable for applications in which temperature rates are not predictable.

To relate the LCST values with the ones reported in the literature for similar polymers, the transition temperature values were also calculated in liquid water. For the NIPAAm-based polymer, the LCST was found at $29{ }^{\circ} \mathrm{C}$, whereas for the DEAAm-based polymer, the value was found at $35^{\circ} \mathrm{C}$ (data not shown). These values are in line with what was reported in the literature for similar iCVD polymers. ${ }^{21}$ Differences between the LCST in humidity and liquid water point out that the kinetic processes and chemical rearrangements the polymers undergo with temperature are also influenced by the physical state of the water molecules. Water vapor more easily starts to permeate into the polymer-free volume, as the fastest change in thickness for both polymers is shifted at lower temperatures. Moreover, the LCST of DEAAm-based polymers is known to occur at higher temperatures than NIPAAm-based ones because of the higher hydrophilicity of the monomer moieties. While this behavior was found in liquid water, the LCST found in humidity is comparable to one of the NIPAAm-based polymer, although the complete phase transition occurs in a wider range of temperatures, as above-described. Further insights into the water permeation kinetics are needed to justify this behavior.
Taking into account the polymer swelling/deswelling adopting the three different temperature profiles, it can be concluded that the hysteresis is a function of the heating rate. If the heating rate is higher than the polymer chain rearrangement, a surface denser skin-like layer is formed, blocking the out-diffusion of water from the polymer network. This phenomenon was found to decrease at lower heating rates. Furthermore, the deswelling was found to be the function of the number of swelling/deswelling cycles the polymer undergoes, indicating a temporary and reversible rearrangement of the polymeric chains, which influence the water out-diffusion.

Starting from the swelling in equilibrium conditions reported in Figure 8a,b, the van't Hoff equation can be adopted to gather information on the enthalpy of swelling for these polymers when in contact with water vapor. The van't Hoff plot for both NIPAAm-based and DEAAm-based polymers is presented in Figure 9.

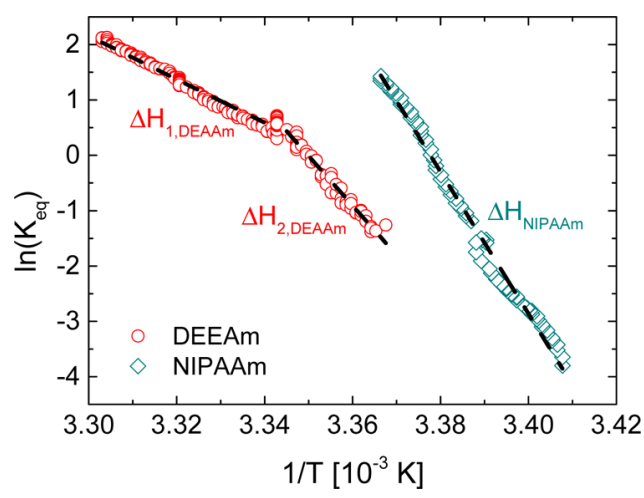

Figure 9. van't Hoff plot for the p(NIPAAm-co-DEGDVE) and $\mathrm{p}$ (DEAAm-co-DEGDVE) in the temperature range where the phase transition occurs. The plot is based on the equilibrium swelling data presented in Figure 8.

The equilibrium constant $K_{\mathrm{eq}}$ is equal to the ratio of collapsed/swollen states. Therefore, it was assumed that at 20 ${ }^{\circ} \mathrm{C}$, all states are in the swollen regime (i.e., $K_{\mathrm{eq}}=0$ ) and that the whole polymer network changed to a collapsed state at 50 ${ }^{\circ} \mathrm{C}\left(K_{\mathrm{eq}}=1\right)$.

For the investigated $\mathrm{p}$ (NIPAAm-co-DEGDVE), $\Delta H$ values were calculated when the phase change occurred and found equal to $990 \pm 50 \mathrm{~kJ} / \mathrm{mol}$. A positive enthalpy is a sign of an endothermic process, as expected. In the literature, pure $\mathrm{iCVD}$ pNIPAAm showed higher enthalpy values, on the order of 1650 $\mathrm{kJ} /$ mol-coop. unit, whereas the other cross-linked p(NIPAAmco-DEGDVE) obtained by $\mathrm{iCVD}$ have shown similar values 
(810 kJ/mol-coop. unit). ${ }^{17}$ Both values were calculated for swelling of the polymer in liquid water. Differences from pure NIPAAm-based polymers are expected because of the absence of cross-linking units, which limits the swelling and decrease in the number of available NIPAAm units in the polymer. For similar cross-linked systems, enthalpy values were calculated with respect to the moles of cooperative unites, that is, active NIPAAm moieties included in the polymer backbone. The values here reported are instead expressed with respect to moles of both cross-linker and cooperative NIPAAm units. Notwithstanding the lower swelling ratio reached with our layers compared to the ones reported in ref 17 , the quicker response to temperature variation, limited to $1.5^{\circ} \mathrm{C}$ (see Figure 8a) for a complete phase transition, compensates for the lower water uptake, accounting for the enthalpy values.

For the $\mathrm{p}$ (DEAAm-co-DEGDVE), two distinct slopes were found in the van't Hoff plot (see Figure 9). At lower temperature, a first swelling mechanism was witnessed, showing values of $\Delta H$ lower but relatively close to the one of the NIPAAm-based polymer $\left(\Delta H_{2, \text { DEAAm }}=750 \pm 10 \mathrm{~kJ} / \mathrm{mol}\right)$. The phase transition occurs at $24.8^{\circ} \mathrm{C}$, that is, a higher temperature compared to $21.7{ }^{\circ} \mathrm{C}$ observed for the NIPAAm-based polymers, as reported in Table 1 for the temperature profile 3. At higher temperature, a second swelling mechanism was found to occur (Figure 9, $\Delta H_{1, \text { DEAAm }}$ ). This mechanism has a clear slower kinetic, with $\Delta H$ values of $325 \pm 20 \mathrm{~kJ} / \mathrm{mol}$. The DEAAm-based polymers showed an extended phase transition, occurring within $5{ }^{\circ} \mathrm{C}$. The slower kinetic profile can be attributed to the lack of free $\mathrm{N}-\mathrm{H}$ moieties.

\section{CONCLUSIONS}

Thermoresponsive hydrogel thin films have been synthesized via iCVD. This study sheds a light into the applicability of these systems, as humidity sensors in real applications. In situ spectroscopic ellipsometry measurements have shown that the obtained hydrogels strongly respond to changes in temperature in relative humidity $>90 \%$. Thickness changes up to $120 \%$ were found when the system undergoes the reversible phase transition at its LCST. Two different hydrogels, $p$ (NIPAAmco-DEGDVE) and $\mathrm{p}$ (DEAAm-co-DEGDVE), with different degrees of cross-linking were studied. The system showed fast response to changing temperatures. A hysteresis was found in the swelling/deswelling as a function of temperature, number of swelling/deswelling cycles, and temperature change rate. The LCSTs differ by $15{ }^{\circ} \mathrm{C}$ for temperature ramps with a shorter time scale (heating/cooling from 20 to $50{ }^{\circ} \mathrm{C}$ in $5 \mathrm{~min}$ ), which decreased to $10^{\circ} \mathrm{C}$, when the temperature that a profile with a ramping time of $10 \mathrm{~min}$ was applied. This hysteresis decreased further by using a different temperature ramp (change of $2{ }^{\circ} \mathrm{C}$ every $2 \mathrm{~min}$ ), where the LCSTs appear within $5{ }^{\circ} \mathrm{C}$. It was found that the deswelling process is happening in a two-step manner with a longer time scale than the swelling process for both materials, whereas the swelling during cooling appears to be one single process. An explanation for this two-step phenomenon is the creation of a thin dense skin in the beginning of the deswelling process, which prohibits the water to leave the system. Furthermore, different cycles of swelling and deswelling showed differences in the mechanisms of water uptake/expulsion, pointing out structural variations of the polymer as a function of cycles. The DEAAm-based polymers did not show any change in the LCST values as a function of the temperature change rate, whereas the NIPAAm-based systems did. This characteristic is crucial when designing sensors based on thin film NIPAAm-based polymers, as the temperature rate variation should be limited to have a precise response of the thermoresponsive polymer. On the contrary, notwithstanding the slower response to temperature variations and the limited change in volume, the DEAAm-based polymers showed a more stable LCST, which makes them more suitable for applications in which temperature rates are not predictable.

\section{ASSOCIATED CONTENT}

\section{S Supporting Information}

The Supporting Information is available free of charge on the ACS Publications website at DOI: 10.1021/acsami.7b18878.

Comparison between swelling behavior of NIPAAm and DEAAm-based polymers (PDF)

\section{AUTHOR INFORMATION}

\section{Corresponding Author}

*E-mail: anna.coclite@tugraz.at.

\section{ORCID}

Alberto Perrotta: 0000-0002-7007-6153

Anna Maria Coclite: 0000-0001-5562-9744

\section{Notes}

The authors declare no competing financial interest.

\section{ACKNOWLEDGMENTS}

This project has received funding from the European Research Council (ERC) under the European Union's Horizon 2020 research and innovation program (grant agreement 715403).

\section{ADDITIONAL NOTE}

${ }^{a}$ It is worth noting that the monomer fraction here indicates the relative available adsorbed monomer on the surface prior and during the deposition and it is related to, but does not correspond to, the amount of monomer included in the polymeric structure.

\section{REFERENCES}

(1) Ramakrishnan, N.; Vamsi, T.; Khan, A.; Nemade, H. B.; Palathinkal, R. P. Humidity Sensor Using NIPAAm Nanogel as Sensing Medium in SAW Devices. Int. J. Nanosci. 2011, 10, 259-262.

(2) Palagi, S.; Mark, A. G.; Reigh, S. Y.; Melde, K.; Qiu, T.; Zeng, H.; Parmeggiani, C.; Martella, D.; Sanchez-Castillo, A.; Kapernaum, N.; Giesselmann, F.; Wiersma, D. S.; Lauga, E.; Fischer, P. Structured Light Enables Biomimetic Swimming and Versatile Locomotion of Photoresponsive Soft Microrobots. Nat. Mater. 2016, 15, 1-8.

(3) Fusco, S.; Huang, H.-W.; Peyer, K. E.; Peters, C.; Häberli, M.; Ulbers, A.; Spyrogianni, A.; Pellicer, E.; Sort, J.; Pratsinis, S. E.; Nelson, B. J.; Sakar, M. S.; Pané, S. Shape-Switching Microrobots for Medical Applications: The Influence of Shape in Drug Delivery and Locomotion. ACS Appl. Mater. Interfaces 2015, 7, 6803-6811.

(4) Chu, L.-Y.; Xie, R.; Ju, X.-J.; Wang, W. Smart Hydrogel Functional Materials; Springer Berlin Heidelberg: Berlin, 2013.

(5) Hoffman, A. S. Stimuli-Responsive Polymers: Biomedical Applications and Challenges for Clinical Translation. Adv. Drug Delivery Rev. 2013, 65, 10-16.

(6) Birgersson, E.; Li, H.; Wu, S. Transient Analysis of TemperatureSensitive Neutral Hydrogels. J. Mech. Phys. Solids 2008, 56, 444-466.

(7) Alf, M. E.; Godfrin, P. D.; Hatton, T. A.; Gleason, K. K. Sharp Hydrophilicity Switching and Conformality on Nanostructured Surfaces Prepared via Initiated Chemical Vapor Deposition (iCVD) of a Novel Thermally Responsive Copolymer. Macromol. Rapid Commun. 2010, 31, 2166-2172. 
(8) Schild, H. G.; Tirrell, D. A. Microcalorimetric Detection of Lower Critical Solution Temperatures in Aqueous Polymer Solutions. J. Phys. Chem. 1990, 94, 4352-4356.

(9) Nguyen, M.; Felidj, N.; Mangeney, C. Looking for Synergies in Molecular Plasmonics through Hybrid Thermoresponsive Nanostructures. Chem. Mater. 2016, 28, 3564-3577.

(10) Matsuo, E. S.; Tanaka, T. Kinetics of Discontinuous VolumePhase Transition of Gels. J. Chem. Phys. 1988, 89, 1695-1703.

(11) Andersson, M.; Axelsson, A.; Zacchi, G. Swelling Kinetics of Poly(N-Isopropylacrylamide) Gel. J. Controlled Release 1998, 50, 273281.

(12) Gan, D.; Lyon, L. A. Tunable Swelling Kinetics in Core-Shell Hydrogel Nanoparticles. J. Am. Chem. Soc. 2001, 123, 7511-7517.

(13) Kaneko, Y.; Sakai, K.; Kikuchi, A.; Sakurai, Y.; Okano, T. Fast Swelling/deswelling Kinetics of Comb-Type Grafted poly(N-Isopropylacrylamide) Hydrogels. Macromol. Symp. 1996, 109, 41-53.

(14) Park, T. G.; Hoffman, A. S. Deswelling Characteristics of Poly(N-Isopropylacrylamide) Hydrogel. J. Appl. Polym. Sci. 1994, 52, $85-89$.

(15) Rice, C. V. Phase-Transition Thermodynamics of NIsopropylacrylamide Hydrogels. Biomacromolecules 2006, 7, 29232925.

(16) Secrist, K. E.; Nolte, A. J. Humidity Swelling/Deswelling Hysteresis in a Polyelectrolyte Multilayer Film. Macromolecules 2011, 44, 2859-2865.

(17) Alf, M. E.; Hatton, T. A.; Gleason, K. K. Insights into Thin, Thermally Responsive Polymer Layers through Quartz Crystal Microbalance with Dissipation. Langmuir 2011, 27, 10691-10698.

(18) Lau, K. K. S.; Gleason, K. K. Initiated Chemical Vapor Deposition (iCVD) of Poly (Alkyl Acrylates): An Experimental Study. Macromolecules 2006, 39, 3688-3694.

(19) Unger, K.; Salzmann, P.; Masciullo, C.; Cecchini, M.; Koller, G.; Coclite, A. M. Novel Light-Responsive Biocompatible Hydrogels Produced by Initiated Chemical Vapor Deposition. ACS Appl. Mater. Interfaces 2017, 9, 17408-17416.

(20) Alf, M. E.; Hatton, T. A.; Gleason, K. K. Novel NIsopropylacrylamide Based Polymer Architecture for Faster LCST Transition Kinetics. Polymer 2011, 52, 4429-4434.

(21) Pena-Francesch, A.; Montero, L.; Borrós, S. Tailoring the LCST of Thermosensitive Hydrogel Thin Films Deposited by iCVD. Langmuir 2014, 30, 7162-7167.

(22) Reeja-Jayan, B.; Kovacik, P.; Yang, R.; Sojoudi, H.; Ugur, A.; Kim, D. H.; Petruczok, C. D.; Wang, X.; Liu, A.; Gleason, K. K. A Route Towards Sustainability Through Engineered Polymeric Interfaces. Adv. Mater. Interfaces 2014, 1, 1400117.

(23) Gleason, K. K. CVD Polymers: Fabrication of Organic Surfaces and Devices; John Wiley \& Sons, 2015.

(24) Alf, M. E.; Hatton, T. A.; Gleason, K. K. Initiated Chemical Vapor Deposition of Responsive Polymeric Surfaces. Thin Solid Films 2011, 519, 4412-4414.

(25) Schmaljohann, D.; Beyerlein, D.; Nitschke, M.; Werner, G. Thermo-Reversible Swelling of Thin Hydrogel Films Immobilized by Low-Pressure Plasma. Langmuir 2004, 20, 10107-10114.

(26) Makino, K.; Hiyoshi, J.; Ohshima, H. Kinetics of Swelling and Shrinking of Poly (N-Isopropylacrylamide) Hydrogels at Different Temperatures. Colloids Surf., B 2000, 19, 197-204.

(27) Unger, K.; Resel, R.; Coclite, A. M. Dynamic Studies on the Response to Humidity of Poly (2-Hydroxyethyl Methacrylate) Hydrogels Produced by Initiated Chemical Vapor Deposition. Macromol. Chem. Phys. 2016, 217, 2372-2379.

(28) Wang, X.; Qiu, X.; Wu, C. Comparison of the Coil-to-Globule and the Globule-to-Coil Transitions of a Single Poly(N-Isopropylacrylamide) Homopolymer Chain in Water. Macromolecules 1998, 31, 2972-2976.

(29) Cheng, H.; Shen, L.; Wu, C. LLS and FTIR Studies on the Hysteresis in Association and Dissociation of Poly(N-Isopropylacrylamide) Chains in Water. Macromolecules 2006, 39, 2325-2329. 